\title{
Land versus pool based physiotherapy in fibromyalgia
}

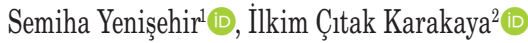 \\ ${ }^{1}$ Department of Physiotherapy and Rehabilitation, Muş Alparslan University, Faculty of Health Sciences, Muş, Turkey \\ ${ }^{2}$ Department of Physiotherapy and Rehabilitation, Muğgla Sitkı Koçman University, Faculty of Health Sciences, Muğgla, Turkey \\ Received: December 04, 2019 Accepted: December 31, 2019 Published online: March 03, 2020
}

We read the article by Acosta-Gallego et al. ${ }^{[1]}$ published in your journal and congratulate the authors for conducting and reporting the findings of a longterm study ( 20 weeks) on the effects of pool versus land based physiotherapy interventions in female patients with fibromyalgia. However, we would like to offer the following commentary to contribute to the interpretation of the findings and be taken into consideration in the future studies on this topic.

The authors lack to provide sufficient background for their research purpose. It is not clear why they thought that land and pool environments might have different effects on the investigated outcomes, if there are other studies which previously compared the effects of these environments, and how this study might contribute to the current literature knowledge.

Furthermore, we see that the source of the subjects was not reported, key outcomes were only measured in less than $85 \%$ of subjects initially allocated to groups (inadequate follow-up), and no intention-to-treat analysis was performed. The six-minute walk test parameters (distance and time) appear to be used to predict the maximal oxygen uptake values, and it is not clear why and how heart rate monitor values were used. Explanation about visual analog scale scoring is also not satisfactory, and we do not know if these scores reflect intensity of pain during resting or any activity. Although the structural stages of the physiotherapy programs and the warm-up activities (walking) were defined, the main exercise stage was described inexplicitly. Which specific exercises were used, and how progressive load principles were applied? Which stretching exercises and relaxation methods were used, and for which muscles? These unclear issues preclude clinicians to repeat the same procedures to achieve the mentioned benefits of the physiotherapy.

Although Table 1 was referenced for the sociodemographic and clinical findings of the participants, this table only covers the physical characteristics; therefore, we do not know if the groups were really similar in regards to sociodemographic and clinical characteristics. The results section only gives pre-post differences of the outcome measures, and we do not know if the groups were similar for the baseline characteristics. There is lack of information about the use of any medications during the long-lasting study period, so we cannot be sure that the achieved improvements are due only to the interventions used. In addition, lack of assessor blindness might have resulted in biased ascertainment of outcomes.

When these methodological limitations are taken into consideration, it is considered that the internal validity (believability) and interpretability, thus the quality of this study is limited. ${ }^{[2]}$ These methodological shortcomings might have masked or overstated the actual effectiveness of both interventions, so the findings should be verified through high-quality studies.

\section{Declaration of conflicting interests}

The authors declared no conflicts of interest with respect to the authorship and/or publication of this article.

\section{Funding}

The authors received no financial support for the research and/or authorship of this article. 


\section{REFERENCES}

1. Acosta-Gallego A, Ruiz-Montero PJ, Castillo-Rodríguez A. Land-and pool-based intervention in female fibromyalgia patients: A randomized-controlled trial. Turk J Phys Med Rehab 2018;64:337-43.

2. Fitzpatrick RB. PEDro: A Physiotherapy Evidence Database. Med Ref Serv Q 2008;27:188-97.

This letter was submitted to the authors of the manuscript; however, not responded. 